\title{
Impact De La Fiscalite Sur La Croissance Economique Du Maroc
}

\author{
Moustapha Hamzaoui \\ Professeur de Sciences Economiques, \\ Université Abdelmalek Essaadi, Faculté de Droit-Tanger \\ Nezha Bousselhami \\ Inspecteur Divisionnaire des Impôts-Doctorante, \\ Université Abdelmalek Essaadi, Faculté de Droit-Tanger
}

doi: 10.19044/esj.2017.v13n4p104 URL:http://dx.doi.org/10.19044/esj.2017.v13n4p104

\begin{abstract}
This paper examines how the taxation (tax revenue) affects the economic growth in Morocco relying to the endogenous growth model of Barro (1990). After recalculating a new series of public capital and private capital and based on simultaneous equations model, one production function type Cobb Douglas with 3 factors (public capital, private capital and employment) has been estimated with data covering the period 1980-2015. The idea is to measure the effect of taxation on economic growth through its impact on public capital. The results find that the relationship between the two variables is positive. The householders can finance the public capital by taxes. And the public capital improves the economic growth.
\end{abstract}

Keywords: Taxation, endogenous growth, public capital, simultaneous equations model

\section{Résumé}

L’objectif du présent article est d'analyser le rôle de la fiscalité dans la croissance économique du Maroc en s'appuyant sur le modèle de croissance endogène de Barro (1990). Après avoir construit des séries de capital public et de capital privé et en se basant sur un modèle à équations simultanées, une fonction de production, de type Cobb Douglas à trois facteurs (capital public, capital privé et emploi), a été estimée sur la période 1980-2015.

L'idée consiste à mesurer l'effet de la fiscalité sur la croissance économique, à travers son impact sur le capital public. Les résultats obtenus vont dans le sens d'une relation croissante entre la fiscalité et la croissance économique 
au Maroc. Ainsi, à travers l’impôt, les ménages contribuent au financement du capital public qui conduit in fine à améliorer la croissance économique.

Mots clés: Fiscalité, croissance endogène, capital public, modèle à équations simultanées

\section{Introduction}

Le développement économique a déjà fait l’objet d’une littérature abondante. Après une analyse souvent savante du sous développement, les spécialistes traitent des voies et moyens du développement. La fiscalité est donnée comme l'un de ces moyens ${ }^{19}$; certains la considèrent même comme «l'une des clefs du développement» ${ }^{20}$

Au niveau théorique, la relation causale entre l'impôt et la croissance économique est souvent appréhendée dans le sens d'une influence de la croissance des variables macro-économiques sur les rentrées fiscales ${ }^{21}$, et rares sont les modèles qui accordent une place importante à la variable fiscale comme facteur déterminant de la croissance ${ }^{22}$. Le rôle de l'impôt dans la détermination du taux de croissance est de ce fait peu pris en considération.

Dépassant la simple technique de couverture des dépenses publiques, la fiscalité est un puissant instrument de politique économique, capable de tendre vers l'optimum économique si elle est correctement conçue ${ }^{23}$. Dans le cas contraire, elle risque d'entraîner des pertes nettes de production, de productivité et d'utilité sociale (RAJHI, 1993) ${ }^{24}$. L'intervention par l'impôt s'avère, de ce point de vue et, en conséquence, nécessaire pour éviter les crises économiques, stimuler le processus de croissance et réduire les inégalités sociales ${ }^{25}$.

Cette liaison est-elle vérifiée pour le cas du Maroc? En vue de répondre à cette question, le présent travail sera organisé de la manière suivante : dans une première section nous allons présenter le soubassement théorique d'une telle liaison, puis, dans la deuxième section, un modèle à équations simultanées sera estimé, pour tester empiriquement le lien entre les deux composantes.

Les résultats obtenus vont confirmer l'impact positif de la fiscalité sur la croissance économique du Maroc. Les individus participent au

\footnotetext{
${ }^{19}$ OCDE, 2009.

${ }^{20}$ Idem.

${ }^{21}$ HENIN.P.Y et RALLE.P, 1993.

${ }^{22}$ CHARLOT.S et SCHMIT.B, 1999.

${ }^{23}$ VALENDUC.C, 2011.

${ }^{24}$ Cité par HENIN.P.Y et RALLE.P, 1993.

${ }^{25}$ VALENDUC.C, 2011
} 
financement du capital public par le biais de l’impôt. Ce capital public contribue, à son tour, à la croissance économique.

\section{Liaison entre fiscalité et croissance économique}

Un grand nombre de travaux sont consacrés à l'analyse empirique des facteurs de la croissance économique, mais ils omettent le plus souvent de tenir compte de la fiscalité ${ }^{26}$. Par cet article nous voulons pallier cette lacune par une estimation économétrique sur données marocaines; mais avant de passer à la modélisation, il serait utile de présenter, dans cette section du travail, le soubassement théorique sur lequel on s'est basé pour construire notre modèle. Dans une première sous-section, nous allons aborder les développements de théories de la croissance endogène. La deuxième soussection évoquera le lien théorique entre la fiscalité et la croissance. Tandis que la troisième va être consacrée à la revue de la littérature empirique.

\section{Développements des théories de la croissance endogène}

Les modèles de croissance endogène constituent le nouvel empire de la théorie de la croissance. Ils justifient leur existence dans la recherche de déterminants économiques qui expliqueraient le rythme de progrès technique, exogène dans le modèle fondateur de Solow. Trois grandes figures dominent les théories de la croissance endogène : Paul M. ROMER, Robert E. LUCAS et Robet J. BARRO.

Les principales directions de ces théories sont : l'accumulation des connaissances et du capital technologique, l'accumulation du capital humain et l'accumulation en dépenses publiques.

ROMER considère que l'accumulation des connaissances est un facteur endogène de croissance. Il reprend en partie la théorie du « learning by doing " déjà formulée par ARROW (1962) qui considère que c'est en produisant qu'une entreprise acquiert des expériences et donc des connaissances. Plus la croissance est forte, plus l'accumulation d'expériences et de savoir faire est forte, ce qui favorise la croissance et ainsi s'installe le cercle vertueux. L'accumulation des connaissances produit des externalités positives. Une entreprise accumule des connaissances qui lui permettent d'être plus performante, mais qui serviront aussi aux autres firmes, par effet d'imitation ou grâce au « turn-over » d'une main d'œuvre ayant bénéficié du savoir faire. L'accumulation des connaissances a donc une productivité privée (celle dont profite l'entreprise), mais également une productivité sociale (celle dont profite l'ensemble de l'économie et de la société). Alors que l'apprentissage par la pratique est à l'origine du progrès technique, ROMER (1996) estime que le taux d'accumulation des connaissances ne

\footnotetext{
${ }^{26}$ BRUN.J.F, CHAMBAS.G, COMBES.J.L; 1998.
} 
dépend pas seulement de la part des ressources que l'économie engage dans la recherche et développement $(\mathrm{R} \& \mathrm{D})$, mais également de l'importance de nouvelles connaissances engendrées par l'activité économique elle-même. C’est donc l'innovation et la R\&D qui constituent le facteur résiduel : plus les efforts de R\&D sont importants, plus la croissance est forte, et inversement.

Le premier modèle de croissance endogène avec capital humain est formulé par LUCAS (1988), qui, plutôt que d'introduire le stock des connaissances sous forme d'externalités, comme ROMER (1986), considère des connaissances accumulatives et appropriables privativement, procurant une incitation individuelle à l'éducation. Ceci dans une inspiration proche du modèle de capital humain de BECKER (1964). La croissance économique dépend donc en grande partie des efforts en formation individuels et sociaux, qui eux-mêmes dépendent de la capacité à épargner et donc de renoncer à une consommation présente pour investir dans l'éducation. Ainsi, la croissance est endogène et cumulative car la capacité en épargne de la formation d'une économie dépend du niveau de production et donc de la croissance économique. Par effet de réseau, un niveau d'éducation est d'autant plus efficace qu'il permet d'interagir avec d'autres personnes présentant ce même niveau. On conçoit alors clairement que la productivité sociale de la formation est supérieure à sa productivité privée.

Bien que les pères fondateurs de la théorie de la croissance endogène, à savoir ROMER et LUCAS, rejettent le rôle primordial de l'Etat, ils acceptent cependant que l'Etat doit favoriser la croissance de longue période. La question n'est pas de savoir si l'Etat doit intervenir ou non dans l'activité économique, mais de savoir comment et jusqu’où peut-il intervenir.

En 1990, BARRO a démontré que le moteur de croissance endogène est l'investissement public en infrastructures. Les dépenses d'infrastructures deviennent un facteur de production à part entière, une source d’accumulation. Cela rejoint des préoccupations anciennes sur la nécessité de promouvoir des réseaux d'infrastructures de qualité pour favoriser la croissance.

Les dépenses d’infrastructures augmentent la productivité du capital physique privé (en permettant des gains de temps, en réduisant les coûts de production) et constituent donc un facteur de production externe à la firme : les firmes ne prennent pas en compte, dans leurs plans d'optimisation, l'accumulation de capital public que leur activité engendre (en effet: une utilisation accrue de capital physique augmente sa production et donc les impôts versés à l’Etat et permet à celui-ci d'améliorer les infrastructures).

La présence de cette externalité explique que l'équilibre concurrentiel décentralisé (sans intervention de l'Etat) aboutit à une croissance sousoptimale. D’où la justification de l'intervention de l'Etat pour rapprocher 
l'économie de l’optimum, en internalisant, à la place des agents privés, l'effet externe. On s’éloigne donc de la justification Keynésienne de l'utilisation des dépenses publiques pour relancer la demande à CT. Ici les dépenses publiques, tournées vers les infrastructures, sont utilisées pour favoriser la croissance de l’offre à LT.

Le modèle de BARRO considère non pas le stock d'infrastructures publiques mais le flux de dépenses associé. D’où le développement de la fonction Cobb-Douglas à trois facteurs (K, L, G) avec rendements d'échelle constants pour $K$ et $L$. Les dépenses $G$ sont financées par un impôt proportionnel aux revenus.

\section{Fiscalité et croissance économique}

L’intervention de l'Etat dans le domaine économique et financier, à la fin du $\mathrm{XIX}^{\mathrm{e}}$ siècle, était très limitée. Son statut d' « Etat gendarme » ${ }^{27}$, le confinait dans les missions de sécurité des citoyens et de défense de la souveraineté du pays. Considérées comme improductives par la théorie libérale classique, les dépenses publiques devraient être strictement limitées. Quant à l'impôt, et si l'on fait référence à la définition de Gaston JEZE, il est « une prestation pécuniaire requise des particuliers, par voie d'autorité, à titre définitif, et sans contrepartie, en vue de la couverture de charges publiques ${ }^{28}$. Cette situation a été résumée par JEZE.G, dans une citation devenue célèbre : «il y a des dépenses, il faut les couvrir » ${ }^{29}$. Depuis 1929, le problème financier a pris une autre dimension. L'Etat, parallèlement à sa fonction classique, qui consiste à financer les services publics, intervient fortement dans la vie économique, pour régulariser la croissance et réduire les inégalités de revenus.

Dans les pays en développement, la faiblesse de l'initiative privée, rend la tâche de l'Etat encore plus ardue. L'Etat se trouve de plus en plus conduit à prendre à sa charge la relance de l'économie. Dès lors, les choix publics acquièrent une importance nouvelle. Les ressources étant limitées et les besoins immenses, il faut, non seulement rationaliser au maximum les choix budgétaires, mais aussi et surtout promouvoir une politique fiscale susceptible de mobiliser le maximum de ressources. Dans ce sens, c'est le niveau des recettes qui détermine le niveau des dépenses. La politique fiscale devient l'une des politiques privilégiées de l'Etat. Une fiscalité fonctionnelle $^{30}$ exige le franchissement d'un seuil minimum de pression fiscale. C'est en fonction de ce seuil que les fiscalistes préconisent une élévation de la pression fiscale dans les pays en développement. Cependant,

\footnotetext{
${ }^{27}$ HORUSITZKY.P, 2013.

${ }^{28}$ JEZE.G, 1936.

${ }^{29}$ CONAN.M, 2008.

${ }^{30}$ KOKKE.M , WEIZIG.F, 2008.
} 
l'augmentation de la pression fiscale dans les jeunes Etats, se heurte à plusieurs limites. Tout d'abord, le faible niveau de la production dans ces pays limite considérablement la capacité contributive des redevables. L’expérience montre que le prélèvement fiscal gagne en importance lorsque le processus de développement et d'industrialisation s'accentue ${ }^{31}$. Ensuite, l'inéquitable répartition de la charge fiscale constitue une seconde limite à l'augmentation de la pression fiscale ${ }^{32}$. La fiscalité dans les PED est d'une manière générale une fiscalité non consensuelle. Enfin, on peut noter l'attitude égoïste et irresponsable des groupes privilégiés, qui ont tendance à faire supporter l'essentiel de la charge fiscale par les classes les plus déshéritées $^{33}$.

Par ailleurs, le prélèvement fiscal dépend du potentiel fiscal de chaque pays; ce dernier varie d'une manière considérable d'un pays à l'autre, en fonction de facteurs structurels relatifs à chaque pays. Une politique fiscale efficace, doit être définie en fonction de la stratégie de développement économique et social choisie. Elle doit avoir pour support un système fiscal juste, souple et adapté et doit viser la réalisation d’objectifs clairs, cohérents et précis.

La complexité des problèmes fiscaux dans les PED nait précisément de l'ambiguïté et de la diversité des fins poursuivies: opérer une redistribution égalitaire du revenu national, accélérer et orienter la croissance économique, augmenter la formation de l'épargne publique etc... Un taux de croissance élevé et une répartition des revenus plus équitable constituent les objectifs officiels de toute "société progressive». Cependant, ces deux objectifs entrent en conflit, l'un avec l'autre. Nous devons alors axer notre choix sur l'un ou l'autre. La question qui se pose alors est de savoir si le prix qu’il faut payer, pour une plus grande égalité, doit se traduire en termes de retards de croissance.

Devant les faibles taux de croissance des économies en développement, la redistribution des revenus apporte relativement peu aux groupes à faible revenu, beaucoup moins que ce que ses défenseurs prétendent $^{34}$. Faire payer les riches est avantageux en terme politique, mais ne l'est guère en termes de pouvoir d'achat. La solution à ce problème épineux relève d'un choix éthique sur l'importance relative à attribuer à la répartition et à la croissance du revenu, dans le court et le long terme. Il semble que dans un premier temps la fiscalité doit encourager et stimuler la croissance économique. Même les groupes, à faibles revenus, ont plus à gagner d'une expansion économique rapide que d'une politique de

\footnotetext{
${ }^{31}$ TANZI.V, ZEE.H, 2001.

${ }^{32}$ Idem.

${ }^{33}$ ITRIAGO.D, 2011

${ }^{34}$ Idem
} 
redistribution des revenus qui risque d'être celle de la pauvreté ${ }^{35}$. L'objectif de correction des disparités collectives, ne peut se réaliser qu'à la suite d'une croissance économique relativement élevée et durable.

La croissance repose sur trois principes: la restriction de la consommation, le désir de produire le plus possible et la préférence pour l'investissement productif. La fiscalité, avec les possibilités d'incitation qu'elle offre, constitue un important stimulant quant à la réalisation des objectifs du plan de développement. Dans cette optique, l'objectif est, d'une part, d'amener les épargnants à investir d'une manière productive, et d'autre part, de réaliser des consommations préférentielles par voie fiscale. C’est le concept même de la fiscalité fonctionnelle, dont les effets ne dépendent pas seulement du volume total des recettes mais également de leurs incidences.

L'objectif de croissance économique place la fiscalité, encore une fois, devant la nécessité d'un choix. S'agit-il de viser l'augmentation de la production de l'entreprise privée, en détournant l'épargne des emplois improductifs ou de faible intérêt pour l'économie, ou alors miser sur l'investissement public et dans ce cas augmenter la formation de l'épargne publique?

Dans les pays libéraux développés, la croissance dépend dans une large mesure du dynamisme de l'entreprise privée. Les insuffisances de cette dernière, dans les pays en développement, ne sont plus à démontrer. Elles proviennent essentiellement du comportement irrationnel des titulaires de hauts revenus. Ceux-ci sont attirés, d'abord, par la thésaurisation (achat d'or), les placements stériles, ou la consommation ostentatoire. Le dynamisme et l'esprit d'entreprise de l'initiative privée est encore, dans les PED, à l'état embryonnaire.

La déficience de l'entreprise privée rend nécessaire une augmentation de l'épargne publique. L'Etat doit alors se substituer à l'entrepreneur privé. Reste à savoir si l'importance qu'on accorde à l'augmentation des recettes publiques ne risque-t-elle pas, d'une part, de freiner l'ascension d'une minorité de riches qui épargnent et investissent dans les secteurs productifs une partie substantielle de leurs revenus et, d'autre part, d'augmenter la consommation publique.

En supposant que les dépenses publiques sont rationalisées au maximum et que les investissements productifs ont la priorité, la fiscalité peut et doit aider à la réalisation des objectifs suivants:

- Mobiliser l'épargne publique et favoriser la formation d'une épargne privée;

- Canaliser l'épargne dans ses utilisations les plus productives telles que l'éducation et la santé;

${ }^{35}$ MOUMMI.A, 2012. 
- $\quad$ Encourager l'investissement productif, national et étranger;

- $\quad$ Atténuer la régressivité de l’imposition indirecte;

- $\quad$ Restreindre la consommation de luxe.

Mais quels que soient le rôle et l'importance que peut revêtir une politique fiscale de développement, elle ne peut être efficace que si elle repose sur une volonté politique de croissance. Un système fiscal n’est pas un simple aménagement des techniques d'imposition, il est avant tout un fait moral et politique et, à ce titre, dépend étroitement de l'orientation politique du régime en place. Donc, une politique fiscale efficace doit être formulée en fonction des objectifs de la croissance économique et être adaptée à l'environnement sociopolitique.

Les PED, bien que présentant certaines similitudes, ne constituent pas un ensemble homogène. Dans certains pays, le secteur de subsistance est largement prépondérant; dans d'autres le secteur monétarisé est en essor; certains pays enfin sont fortement dépendants de l'extérieur, par rapport à d'autres. Une étude fiscale, consacrée aux PED en général, ne peut rester qu'au niveau des généralités et ne permettrait pas, à notre avis, de scruter un système fiscal déterminé, de le cerner dans ses détails, ses insuffisances et ses imperfections spécifiques. Ces études trop générales sont encore moins inopérantes lorsqu'il s’agit de faire des propositions concrètes de réformes car, en la matière, il n’y a pas encore de thérapeutique standard. Pour ces raisons, nous avons choisi de limiter notre étude au seul cas du Maroc.

Au Maroc, la fiscalité étant le pourvoyeur principal des recettes de l'Etat, les recettes fiscales représentent en moyenne $85 \%$ des recettes ordinaires de l'Etat, réalisées entre 2001 et 2015, avec un pic de $90 \%$ en $2008^{36}$. Face à ces chiffres, il semble nécessaire qu'une réflexion sur le système fiscal, en tant que levier du développement économique et de justice sociale, soit menée. Dans cette perspective, il ne suffit plus qu'un impôt serve à réduire les inégalités, ni même qu'il soit fonctionnel au sens où l'entendait Keynes, c'est à dire dans le court terme, il faut bien entendu que cet impôt possède ces qualités, mais il doit encore et surtout exercer une action à long terme, autrement dit, favoriser la croissance, d’où la nécessité de tester empiriquement cette corrélation fiscalité-croissance.

\section{Revue de littérature empirique}

Parallèlement au débat théorique, porté par les nouvelles théories de la croissance, s'est développée une importante littérature empirique visant à examiner les effets du capital public sur la croissance des nations. Les premières études (Ashauer, 1989 et Munnell, 1990) ${ }^{37}$ ont mis en évidence un

\footnotetext{
${ }^{36}$ Données du Ministère de l’Economie et des Finances.

${ }^{37}$ Cité par CHARLOT.S et SCHMITT.B, 1999.
} 
effet positif du capital public sur la production ou la productivité des entreprises, en estimant des fonctions de production à trois facteurs.

Munnell (1992) ${ }^{38}$ a montré que le déclin de la productivité américaine dans les années 1970 était lié en grande partie à la baisse du taux d’investissement en capital public. Son résultat a été obtenu avec une fonction de production (Cobb Douglas, données en log) dont les facteurs sont le capital privé, le capital public non militaire et l'emploi, auxquels il a ajouté un trend et le taux d'utilisation des capacités de production, afin de prendre en compte les variations conjoncturelles. Les données concernent les Etats-Unis de 1949-1985.

Munnell (1993) ${ }^{39}$ a montré -toujours dans le cas des Etats américains- que l'impact du capital public est plus élevé dans l'industrie manufacturière et l'agriculture que dans les services. Conrad et Seitz $(1992)^{40}$ confirment ce résultat pour les régions allemandes (un travail précurseur de Mera, $1973^{41}$, pour les régions japonaises va dans le même sens). Andersson et alii (1990) ${ }^{42}$, révèlent, de leur côté, pour les régions suédoises que les infrastructures routières sont les plus productives. Ce résultat est confirmé par Garcia-Mila et McGuire (1992) ${ }^{43}$ pour les Etats américains.

Ralle (1993) ${ }^{44}$ a étudié la relation entre productivité privée et investissement public. L’étude a été réalisée à partir de données en coupe sur les régressions françaises, aux années 1970-1989. Il a développé notamment un modèle de croissance endogène dans lequel le taux de croissance de la productivité est relié à la part de l'investissement public (Etat et collectivités locales) dans le PIB. L’objectif était de mesurer l'impact de capital public sur l'activité économique, à la fois dans ses aspects positifs (productivité) et négatifs (financement). Il a montré que les régions dont le taux d'investissement public est élevé sont aussi celles où la croissance de la productivité a été la plus rapide. Pour les régions françaises, l’élasticité de la production au capital public est comprise entre 0.02 et 0.09 . Ce résultat montre donc aussi un lien positif et significatif. Il a confirmé aussi si sa diminution lorsque la zone étudiée se réduit.

Ces premiers résultats ont cependant donné lieu à une vive controverse quant à leur robustesse statistique (Eberts, 1990 et Gramlich,

\footnotetext{
${ }^{38}$ Cité par ROY.W, 2004.

${ }^{39}$ Cité par VEGANZONES.M.A, 2000.

${ }^{40}$ Idem.

${ }^{41}$ Idem.

${ }^{42}$ Idem.

${ }^{43}$ Idem.

${ }^{44}$ Cité par ROY.W, 2004.
} 
1994) ${ }^{45}$. Le débat a, d'une part, porté sur l'absence de mesure des effets d'éviction qu'engendrent les investissements publics et sur le sens de relation observée statistiquement. Il a, d’autre part, porté sur les méthodes économétriques mises en œuvre, ces discussions méthodologiques débouchant sur une révision à la baisse des résultats des premiers travaux.

Concernant le sens de causalité de la relation, si le capital public peut être productif, il est financé par les contribuables via l'impôt, la dotation en infrastructures va donc dépendre du revenu de ceux-ci et donc de la production. Il y a alors un effet en retour qu'il est nécessaire de prendre en compte pour mesurer l'effet net du capital public sur la croissance. La correction d'un tel biais de simultanéité s'effectue classiquement en ayant recours à des systèmes d'équations simultanées (Duffy-Deno et Eberts, 1991; Ford et Poret, 1991) ${ }^{46}$.

Cette méthode a été appliquée pour le cas de la France, (Shmitt et Charlot; 1999) en mobilisant des séries régionalisées de capital public et privé et l'économétrie des données de panel, sur la période 1982-1993 et pour les 22 régions françaises. L’introduction de la forme trans-log a permis de calculer les élasticités propres à chaque région et a montré que l'effet positif du capital public est d'autant plus important que la région concernée est riche. Ainsi, si le capital public, en France, était un élément stimulant la croissance régionale, il n’a pas pu jouer un rôle dans la réduction des disparités interrégionales.

En s’inspirant de ces nouvelles théories de croissance endogène, ainsi que de tous ces travaux empiriques, surtout du modèle de Barro qui a intégré la variable fiscale, que nous voulons en tester l'impact sur la croissance économique au Maroc et ce, via son impact sur le capital public, notre modèle va introduire le capital public, le capital privé, le capital humain et la fiscalité.

\section{Effets de la fiscalité sur la croissance économique du Maroc.}

Dans cette section nous allons passer à la modélisation pour tester empiriquement la liaison entre la fiscalité et la croissance économiques au Maroc. Pour ce faire, elle sera organisée en quatre sous sections. La première sous section va aborder la spécification du modèle à équations simultanées, la deuxième va présenter la construction des variables du stock de capital public ainsi que celui du capital privé. La description des données va être présentée dans la troisième sous section. La dernière sera consacrée à l'estimation et l'analyse des résultats.

\footnotetext{
${ }^{45}$ Cité par CHARLOT.S et SCHMITT.B, 1999.

${ }^{46}$ Idem.
} 


\section{Modèle à équations simultanées}

En nous appuyant sur les résultats théoriques des modèles de croissance endogène avec externalités de capital public ${ }^{47}$, nous cherchons à analyser le rôle de la mobilisation des ressources fiscales dans la croissance économique au Maroc. Ces modèles s’appuient le plus souvent sur des fonctions de production à trois facteurs dont deux sont des facteurs privés (travail et capital privé) et le troisième, capital public, est à financement public. La forme fonctionnelle, la plus fréquemment utilisée, est la forme Cobb-Douglas. Cette dernière permet une lecture directe des élasticités et des rendements d'échelle et une discussion aisée de la présence ou non d'externalités de capital public. Il y a en effet externalités engendrées par ce facteur si les rendements d'échelle sont décroissants ou constants en facteurs privés et croissants sur l'ensemble des facteurs, privés et publics ${ }^{48}$.

La fonction de production à trois facteurs prend la forme :

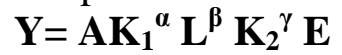

Où $\mathbf{Y}$ est la production mesurée par la valeur ajoutée non agricole, $\mathbf{K}_{\mathbf{1}}$ est le stock de capital public, $\mathbf{L}$ est l'emploi et $\mathbf{K}_{\mathbf{2}}$ est le stock de capital privé. Après la log-linéarisation du modèle précédant et en adoptant le remplacement habituel du logarithme d'une variable $\mathrm{X}$ par une lettre minuscule $x$, le modèle (1) s’écrit comme suit:

$$
\mathbf{y}=\mathbf{a}+\boldsymbol{\alpha \mathbf { k } _ { 1 }}+\boldsymbol{\beta I}+\gamma \mathbf{k}_{2}+\mathbf{e}
$$

Or, le sens de causalité de la relation capital public-production (VA n.a) a été critiqué. En effet, si un pays est riche et productif, il est en mesure de financer un stock de capital public important. C'est alors la richesse du pays qui détermine le stock de capital public et non l'inverse (Herrera, 1996) ${ }^{49}$. Afin d'éviter ce biais de simultanéité, nous allons considérer un modèle à équations simultanées, c'est-à-dire un système dans lequel les variables expliquées sont interdépendantes. Pour cela le taux d'imposition est introduit pour expliquer la capacité du pays à investir en capital public. Ce dernier peut être productif, il est financé par les contribuables via l’impôt, il va donc dépendre du revenu de ceux-ci et donc de la production (VA n.a).

Le système d'équations simultanées prend la forme:

$$
\begin{gathered}
\mathbf{y}=\mathbf{c}_{1}+\mathbf{c}_{2} \mathbf{k}_{1}+\mathbf{c}_{3} \mathbf{k}_{2}+\mathbf{c}_{4} \mathbf{l}+\mathbf{e}_{1} \\
\mathbf{k}_{1}=\mathbf{c}_{5}+\mathbf{c}_{6} \mathbf{y}+\mathbf{c}_{7} \mathbf{T}+\mathbf{e}_{2}
\end{gathered}
$$

Où $\mathbf{T}$ désigne le taux de prélèvement fiscal et $\mathbf{e}_{\mathbf{1}}$ et $\mathbf{e}_{\mathbf{2}}$ des termes d'erreurs indépendants. Ce modèle revient donc à estimer une fonction de production de forme Cobb-Douglas à trois facteurs, dans laquelle la fiscalité affecte la production d'un pays via son impact sur le capital public.

\footnotetext{
${ }^{47}$ BARRO, 1990.

${ }^{48}$ CHARLOT.S et SCHMITT.B, 1999.

${ }^{49}$ Cité par CHARLOT.S et SCHMITT.B, 1999.
} 


\section{Variables stock de capital public et stock de capital privé}

Au Maroc, le stock de capital est global et n’est pas ventilé en stock de capital public et en stock de capital privé. Pour ce faire, et en s’inspirant du document de travail du FMI « Estimation de la Production Potentielle au Maroc » (Weber.A, 2011), nous avons travaillé avec des hypothèses:

\section{Première hypothèse}

Pour estimer le stock de capital public, nous commençons par calculer le stock de capital public pour une année donnée ${ }^{50}$ en divisant les investissements publics par leur taux de croissance moyen (g) et le taux annuel d'amortissement $(\delta)$, dont on suppose qu'il est égal à $4 \%$, une hypothèse standard rencontrée dans la littérature qui existe déjà (par exemple, Billmeier ; 2004 et Konuki ;2008) ${ }^{51}$.

On a supposé que l'investissement public global est égal à la somme de l'investissement de l'Etat, l'investissement des Entreprises et Etablissements Publics Marocains ainsi que l'investissement des Collectivités territoriales.

En ce qui concerne l'investissement des EPP, une série de 1995 jusqu'au 2015, a été obtenue auprès de la DEPP. Et pour pouvoir la compléter depuis 1980, nous avons estimé les valeurs manquantes en divisant la série dont on dispose par la FBCF. Un taux moyen de 24\% a été obtenu. Autrement dit, les investissements des Entreprises et Etablissements publics représentent, en moyenne, 24\% de la FBCF. En multipliant, alors, la FBCF de 1980 à 1994 par 0.24, notre série devient complète.

Même traitement pour l'investissement des CL, la série qu’on a pu avoir de la Trésorerie Générale du Royaume n’est que de 2000 à 2015. Et pour estimer les valeurs manquantes depuis 1980, nous avons divisé la série disponible par la FBCF, un taux moyen de 4\% est obtenu. En appliquant ce taux à la FBCF, de 1980 à 1999, la série devient complète.

$K_{1(1980)}=I_{p u(1980)} /(g+\delta)$,

Avec:

- $\quad \mathbf{K}_{\mathbf{1 ( 1 9 8 0 )}}$ : le stock de capital public pour l’année 1980 ;

- $\quad \mathbf{I}_{\mathbf{p u ( 1 9 8 0 )}}$ : l'investissement public global pour la même année 1980 $=\mathrm{I}($ Etat $)+\mathrm{I}(\mathrm{EEP})+\mathrm{I}(\mathrm{CL})$;

- $\quad$ g : le taux de croissance annuel moyen de l'investissement public sur

la période 1980-2015;

$\begin{array}{ll}\text { valeur } & \boldsymbol{\delta}\end{array} \quad$ 0,04 est le taux de dépréciation étant approximé par la

${ }^{50}$ Dans notre cas, nous commençons par l'année 1980.

${ }^{51}$ Cité dans : « Estimation de la production potentielle au Maroc », FMI 2011. 
appliquée généralement aux pays industrialisés.

\section{Deuxième hypothèse}

Pour obtenir le stock de capital public après 1980, on utilise la formule qui suit :

\section{Troisième hypothèse}

$$
K_{1(t)}=(1-\delta) K_{1(t-1)}+I_{p u(t)}
$$

En suivant le même principe, le stock de capital total relatif à l'année 1980 est obtenu en divisant la FBCF, correspondant à la même année, par leur taux de croissance moyen ( $\mathrm{g}$ ) et le taux annuel d'amortissement $(\delta)$, dont on suppose qu'il est égal à $4 \%$.

Avec:

$$
\mathrm{K}_{1980}=\mathrm{FBCF}_{1980} /(\mathrm{g}+\delta)
$$

- $\quad \mathbf{K}_{\mathbf{1 9 8 0}} \quad$ : le stock de capital total pour l'année 1980.

- $\quad$ FBCF $_{1980}$ : la Formation Brute du Capital Fixe pour la même année 1980.

- $\quad$ g $\quad$ : le taux de croissance annuel moyen de la FBCF sur la période 1980-2015.

- $\quad \delta \quad: 0,04$ est le taux de dépréciation étant approximé par la valeur appliquée généralement aux pays industrialisés.

\section{Quatrième hypothèse}

Le stock de capital total après 1980, est obtenu en utilisant la formule suivante :

\section{Et finalement,}

$$
K_{t}=(1-\delta) K_{t-1}+F B C F_{(t)}
$$

L'obtention du capital privé devient facile, soit :

Avec :

$$
\mathbf{K}_{\mathbf{2 ( t )}}=\mathbf{K}_{\mathbf{t}}-\mathbf{K}_{\mathbf{1}(\mathbf{t})}
$$

- $\quad \mathbf{k}_{2(t)}$ : le stock de capital privé ;

- $\quad \mathbf{k}_{\mathbf{t}} \quad$ : le stock de capital total ;

- $\quad \mathbf{k}_{\mathbf{1}(\mathbf{t})}$ : le stock de capital public.

\section{Description des données}

Après avoir reconstitué des séries sur la variable du stock de capital (public et privé), ces dernières sont représentées dans le graphique suivant : 
Graphique-1: Evolution du stock de capital public et privé au Maroc (1980-2015).

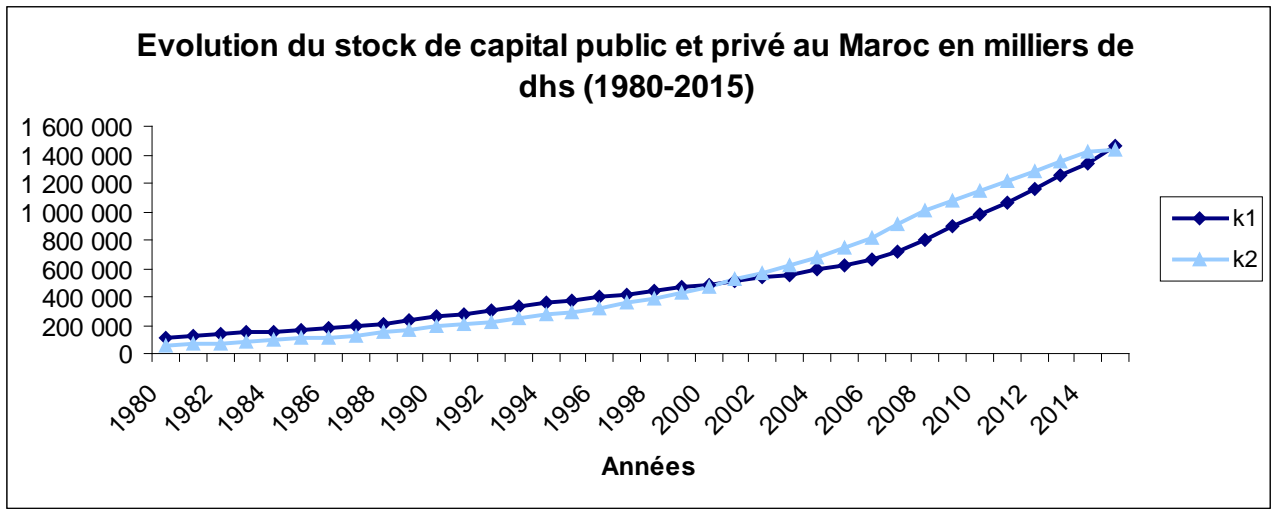

Source de données: Construites par nous mêmes.

D'après le graphique-1 on voit clairement que le stock de capital privé n’a commencé de dépasser celui public qu'en 2000. Chose tout à fait normale, car la fin des années 90 a connu l'ouverture de nombreux secteurs au capital privé y compris le capital privé étranger ${ }^{52}$ (bancaire, assurances, télécommunications, audiovisuel...). L'intensification des efforts de libéralisation économique ainsi que le programme de réformes économique et social, ayant visé le processus d'allègement de procédures administratives pour favoriser l'investissement, ont permis le développement du secteur privé au Maroc, surtout avec le commencement réel des opérations de transfert des entreprises publiques au secteur privé en 1993.

Notre système d'équation contient en plus du logarithme du stock de capital public $\left(\mathrm{k}_{1}\right)$ et du logarithme du celui privé $\left(\mathrm{k}_{2}\right)$ :

- $\quad$ Le logarithme de la production ( $y=\log (V A$ n.a)), prix chaîné base 2007. Les anciennes séries base 1980 et base 1998, publiées par le HCP, ont été recalculées par rétropolation ${ }^{53}$ en base actuellement en vigueur, 2007, pour disposer d'une série homogène longue dans le temps (1980 à 2015).

- Le logarithme de la population active occupée, âgée de 15 ans et Plus, (l): Pour cette variable, il est à signaler qu'on a pu avoir une série annuelle auprès de la DEPF de 1982 jusqu'à 2015. Et puisque nous disposons des séries pour toutes les autres variables depuis 1980, nous avons

\footnotetext{
${ }^{52}$ OUALI.S, 2007: « Réformes et libéralisation des investissements au Maroc », OCDE.

${ }^{53}$ Les changements de base ont pour corollaire d'améliorer les comptes nationaux et de s'adapter mieux aux besoins d'analyse. Ils ont, par contre, l'inconvénient d'entraîner des ruptures dans les séries de comptes. Néanmoins, pour remédier à cette situation et disposer de séries homogènes longues dans le temps, les comptes du passé devraient êtres élaborés en référence à la nouvelle année de base.

Les différentes procédures utilisées pour recalculer les anciens comptes sont dites «la rétropolation des comptes nationaux » de bases précédentes en base actuellement en vigueur.
} 
estimé les deux années manquantes en appliquant le taux de croissance entre 1982 et 1983 puis entre 1983 et 1984, qui est le même et égal à 1,2\%.

- La variable mesurant l'effet de la fiscalité sur la croissance économique (T): C'est le taux de prélèvement fiscal ou le ratio des recettes fiscales sur le PIB non agricole ${ }^{54}$. Les données sur les recettes fiscales sont publiées par le ministère de l'Economie et des Finances et celles relatives au PIB par le HCP. Le graphique-2 trace l'évolution qu'a connu le prélèvement fiscal au Maroc du 1980 au 2015.

Graphique-2: Evolution du taux de prélèvement fiscal (1980-2015).

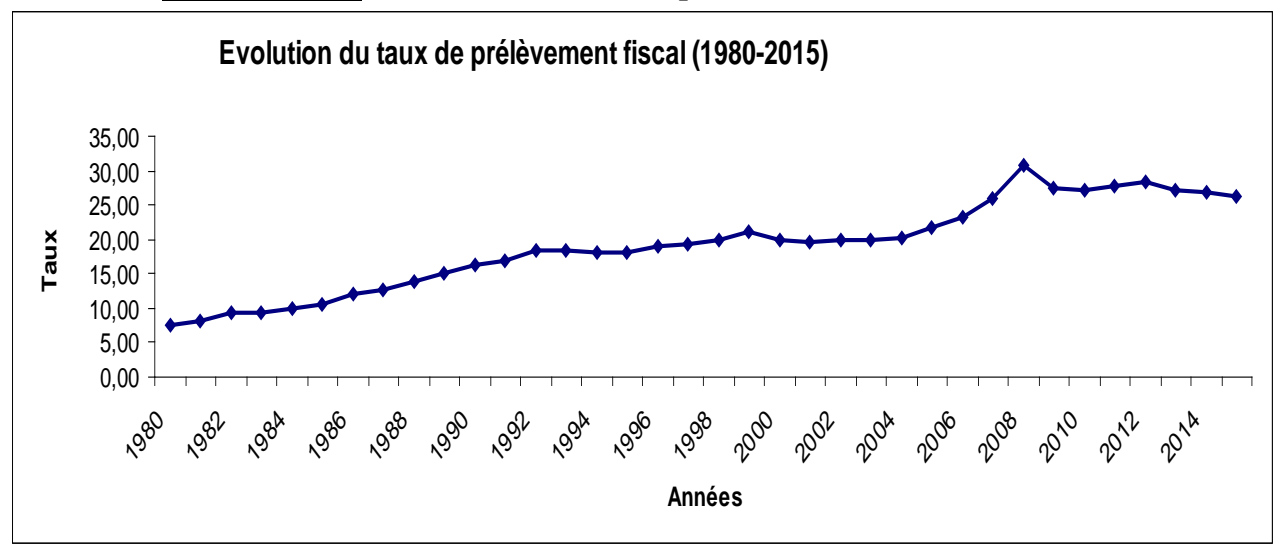

Source de données: Ministère de l’Economie et des Finances et HCP.

L'évolution des recettes fiscales se voit clairement sur le graphique-2. D’un point de vue global, les données portant sur la période 1980-2015font état d'une hausse plus soutenue des recettes fiscales par comparaison au niveau de l'activité. Les recettes fiscales rapportées au PIB non agricole ont, en effet, gagné près de 19 points en pourcentage en passant de $7,66 \%$ en 1980 à 26,35\% en 2015, sans oublier de mentionner la valeur typique enregistrée, en 2008, année de forte croissance, soit 30,82\%. Toutefois, une certaine instabilité du rythme de progression des recettes fiscales a été enregistrée à compter de 2009, s'expliquant, principalement par la conjonction de trois facteurs. Le premier facteur a trait aux effets de la conjoncture économique sur les différents types d'impôts. Ceci est attribuable au ralentissement de l'activité économique suite, notamment, à la forte récession qu'ont connue les principaux partenaires économiques du Maroc au lendemain de la crise économique et financière internationale. Le

\footnotetext{
${ }^{54}$ A signaler que le PIB non agricole est obtenu en retranchant la valeur ajoutée agricole du PIB. (Sachant que les séries de la VA agricole, publiées par le HCP, base 1980 et base 1998, ont été corrigées de la même manière que le PIB précédemment pour pouvoir avoir une série complète de 1980 à 2015, prix chaîné base 2007).
} 
deuxième facteur se rapporte à la réduction des taux de l'IS et de l'IR ${ }^{55}$. Le troisième facteur concerne le démantèlement tarifaire et le comportement baissier des importations taxables qui ont impacté l’évolution des recettes douanières.

Par rapport à de nombreux PED et en dépit de chocs conjoncturels relativement importants (sécheresses, chocs pétroliers,...), le taux de prélèvement fiscal du Maroc est caractérisé, sur la période 1980-2015, par une instabilité moindre, ce qui constitue un facteur favorable à une bonne gestion des finances publiques.

\section{Estimation et analyse des résultats}

L'estimation du modèle a été faite à l'aide du logiciel économétrique Eviews. Sachant que la méthode optée est la MCO, les résultats obtenus sont résumés dans le tableau suivant :

Tableau: Résultats de l'estimation économétrique du modèle à équations simultanées :

fiscalité-k public/k public-production

\begin{tabular}{|c|c|c|c|}
\hline & Coefficient & t-student & prob \\
\hline \multicolumn{4}{|c|}{ Equation1: Production (y) } \\
\hline $\mathbf{a}_{1}$ & 1,469612 & 2,066282 & 0,0428 \\
\hline $\mathbf{K}_{\mathbf{1}}$ & 0,205846 & 2,520556 & 0,0142 \\
\hline $\mathbf{K}_{2}$ & 0,618191 & 11,06054 & 0,0000 \\
\hline $\mathbf{L}$ & 0,844439 & 16,85717 & 0,0000 \\
\hline \multicolumn{4}{|c|}{$R^{2}=0,998792 \quad R^{2}$ ajusté $=0,998679 \quad D W=1,383268$} \\
\hline \multicolumn{4}{|c|}{ Eqaution2: Capital public $\left(\mathrm{K}_{1}\right)$} \\
\hline $\mathbf{b}_{1}$ & 4,997295 & 6,000941 & 0,0000 \\
\hline $\mathbf{Y}$ & 0,890903 & 7,695781 & 0,0000 \\
\hline $\mathbf{T}$ & 0,296158 & 5,008964 & 0,0000 \\
\hline
\end{tabular}

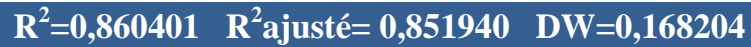

Source : résultats de l'estimation économétrique ${ }^{56}$

Les résultats de cette estimation montrent tout d'abord que le modèle permet d'expliquer dans un premier temps 99\% de la variabilité de la production $\left(\mathrm{R}^{2}\right.$ ajusté $\left.=99 \%\right)$ et dans un deuxième temps $85 \%$ de la variabilité du capital public $\left(\mathrm{R}^{2}\right.$ ajusté $\left.=85 \%\right)$.

Ensuite, à travers la lecture du $\mathrm{T}$ de Student des variables explicatives, nous pouvons déduire que ces dernières sont toutes significatives.

${ }^{55}$ En 2009, le taux marginal de l'IR a été baissé de $42 \%$ à $40 \%$ pour s'établir à $38 \%$ en 2010. Le manque à gagner suite au réaménagement du barème de l'IR était de $0,6 \%$ du PIB en 2009 et 0,5\% en 2010. (Source: Rapport Economique et Financier, 2016). Ajoutant à cela la réduction, en 2008, du taux de l'IS de 35\% à 30\%.

${ }^{56}$ Voir annexe. 
Nos estimations mettent en évidence l'impact significatif sur la production du capital public (élasticité de 0,21). De ce fait, elles valident les résultats des études empiriques citées précédemment surtout celles de Barro (1990), faisant office de référence dans la formulation de l'impact des dépenses publiques productives sur le niveau de l'activité. Autrement dit, l'investissement dans les infrastructures, l'éducation et la santé constitue un facteur favorable à la croissance économique.

De même, le capital privé a un impact largement significatif et positif sur la production (élasticité de 0,62). Ceci prouve l'importance des investissements privés dans l'alimentation d'une croissance économique soutenue à travers la création d'emploi, la progression des revenus et ainsi la génération des recettes indispensables pour que les pouvoirs publics puisse élargir l'accès aux soins de santé, à l'éducation et aux infrastructures, et contribuer ce faisant à améliorer la productivité. Sans négliger l'action bénéfique des dépenses en capital public sur la rentabilité du capital privé. Ceci signifie une complémentarité entre investissement public et investissement privé.

La variable mesurant l'emploi est largement significative. Ce qui signifie que la croissance de la production est impactée par l'importance de la population active occupée. Une hausse de $1 \%$ de cette part serait à la base d'une croissance de l'output de $0,84 \%$.

Les résultats de l'estimation de l'équation du capital public montrent, de leur côté, que celui-ci dépend fortement et positivement du niveau de la croissance économique (élasticité de 0,9), plus le pays a un niveau de production élevé, plus il dépense en capital public. Ce dernier est renforcé, également, par le potentiel fiscal. Les individus participent au financement du capital public par le biais de l'impôt. Le rôle de ce dernier facteur apparait primordial parce que, en attendant une meilleur solution, c'est le seul moyen pratique $^{57}$, pour les pouvoir publics, d'obtenir les ressources dont ils ont besoin pour offrir les produits et services publics exigés par les marocains. Il faut juste un système fiscal efficace et équitable. Autrement dit il faut détecter le taux d'imposition optimal permettant d'arbitrer entre «trop d'impôt tue l'impôt ${ }^{58}$ » et « mieux d'Etat, mieux d'impôts»" ${ }^{59}$.

Le schéma ${ }^{60}$ suivant récapitule toutes les interactions entre ces variables :

\footnotetext{
${ }^{57}$ TANZI.V, ZEE.H, 2001.

${ }^{58}$ Ou Courbe de LAFFER: le taux d'imposition en un premier temps est positivement corrélé à la croissance économique jusqu'un seuil $(\alpha)$. Au-delà de ce seuil correspondant au niveau optimal, toute variation du taux d'imposition est inversement proportionnel au taux de croissance.

${ }^{59}$ Cité par TSASA VANGU.J-P.K, 2012.

${ }^{60}$ Idem.
} 
Schéma -1 : Interactions dépenses publiques et croissance économique.

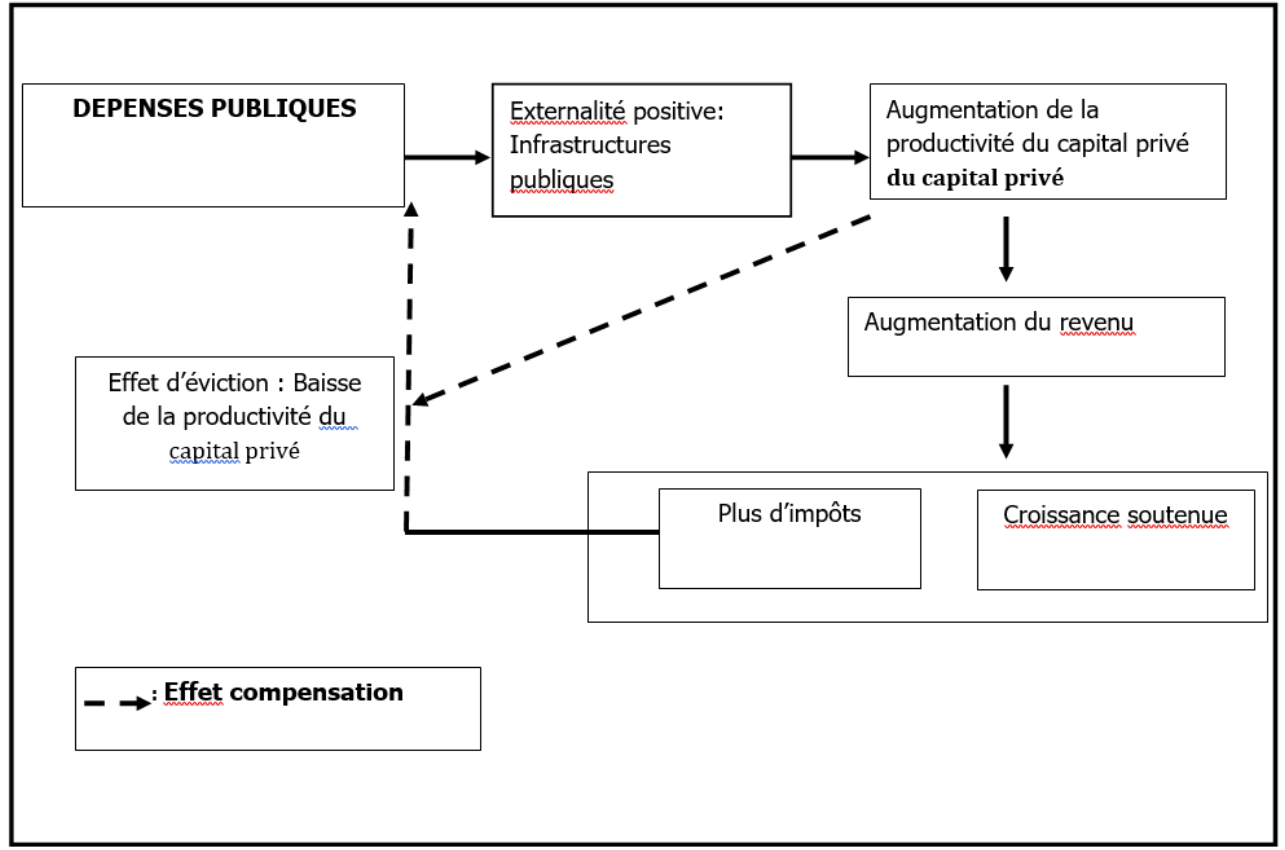

\section{Conclusion}

L'entrée retenue ici pour traiter de la question du rôle de la fiscalité sur la croissance économique est celle d'une fonction de production à trois facteurs, visant à mettre en évidence l'existence d'externalité de capital public. Ce dernier est la variable clé dans notre modèle. Il est dans une première équation, une variable explicative, et dans la deuxième une variable à expliquer. Mises en œuvre sur des séries chronologiques portant sur la période 1980-2015, une telle démarche nécessitait de recourir à un modèle à équations simultanées. Nous avons alors mobilisé les outils de l'économétrie pour tester la forme Cobb Douglas, classiquement utilisée dans ce type d'approche.

Nos résultats mettent tout d'abord en évidence le rôle non négligeable joué par la fiscalité sur la croissance. Autrement dit, les revenus fiscaux peuvent aider les pouvoirs publics à atteindre leurs objectifs économiques et sociaux. Il leur faut, pour ce faire, trouver un juste équilibre pour récompenser l'esprit d'entreprise, l'innovation et la prise de risque, tout en étant capable de financer d'importantes dépenses publiques, notamment des programmes éducatifs et sociaux, ainsi que de grands travaux publics. Toutefois, outre la croissance, le souci de l'équité ainsi que celui de la réduction de la pauvreté doivent être toujours présents, surtout que la fiscalité risque d'entraîner des pertes nettes de production, de productivité et d'utilité sociale. 
Renforcer la mobilisation des ressources nationales ne revient pas uniquement à augmenter les recettes: il s’agit également de concevoir un système de prélèvement propre à favoriser la cohésion et la bonne gouvernance, à améliorer la capacité des pouvoirs publics de rendre compte de leurs décisions aux citoyens et à promouvoir la justice sociale. La conception et le fonctionnement du système de prélèvements, et notamment les dimensions intéressant la transparence, la lutte contre la corruption et l'équité, sont également des facteurs déterminants pour les décisions des investisseurs nationaux et internationaux dans la mesure où ils constituent des paramètres de l'amélioration des conditions d'ensemble pouvant permettre d'attirer davantage d'investissements privés.

\section{References:}

1. AGENOR.P-R, NEANIDIS.K.C, 2015: « Innovation, public capital, and growth », Journal of Macroeconomics, n44, p 252-275, ELSEVIER, April 2015.

2. AKESBI.N, 2008: " Evaluation d'un système fiscal controversé ", Revue Economia, n² fév-mai 2008.

3. AMABLE.B, GUELLEC.D, 1992: « Les théories de la croissance endogène ». Revue d’économie politique, mai-juin, 102 (3).

4. ARROW.K, 1962: "The Economic Implications of Learning by Doing », Review of Economic Studies, 29(2), p 155-173.

5. ARTUS.P, 1993: «Croissance endogènes: revue des modèles et tentatives de synthèse ». Revue économique, 44(2), mars.

6. ARTUS.P, KAABI.M, 1993: «Dépenses publiques, progrès technique et croissance ». Revue économique, 44(2), mars.

7. AUJEAN.M, LORENZI.J.H, 2011: « Fiscalité et croissance », les cahiers : le cercle des économistes. Presses universitaires de France, $1^{\text {ère }}$ Edition, avril, 2011. Descartes \& Cie.

8. AVOUYI-DOVI.S, SASSENOU.M, 1992: «Croissance endogène : une application à l'industrie manufacturière française ».

9. BALDWIN.R.E, 1989: « The Growth Effect of 1992 ». Economic policy, Octobre.

10. BARRO.R, 1990: "Government Spending in a simple model of Endogenous Growth ", journal of political economy, vol.98, N5, S103-S125.

11. BARRO.R, 1991: "Economic Growth in a cross section of countries». Quarterly Journal of Economics, mai.

12. BARRO.R, SALA-I-MARTIN.X, 1992: « Public Finance in Models of Economic Growth ».Review of Economic Studies, 59. 
13. BASSANINI.A, SCARPETTA.S, 2001 : « Les moteurs de la croissance dans les pays de l’OCDE : Analyse empirique des données de panel », Revue Economique de l’OCDE, n³3/II

14. BENSALAH ZEMRANI.A, 2001 : «Les Finances de l'Etat au Maroc, l'entreprises face au fisc », l'Harmattan.

15. BIN.F, 2012 : « Différentes conceptions des réformes fiscales », Gestion et Finances Publiques n ${ }^{\circ} 8-9$.

16. BRUN.J.F, CHAMBAS.G, COMBES.J.L, 1998 : « La politique fiscale agit-elle sur la croissance? », Revue d'Economie du Développement, P: 115-125.

17. BRUN.J.F， CHAMBAS.G， COMBES.J.L， DULLECCO.P, GASTAMBIDE.A, GUERINEAU.S, GUILLAUMONT.S, ROTA GRAZIOSI.G ; 2006 : « Evaluation de l'espace budgétaire des pays en développement » document conceptuel rédigé à la demande du PNUD (CERDI).

18. BRUN.J.F, CHAMBAS .G, COMBES.J.L, 2006 : « Recettes publiques des pays en développement: méthodes d’évaluations ». (CERDI), STATECO, n¹00.

19. BRUN.J.F, CHAMBAS .G, GUERINEAU.S, 2008 : « Aide et mobilisation fiscale dans les pays en développement ». CERDI, Etudes et Documents, E 2008.12.

20. BRUN.J.F， CHAMBAS .G, GRAZIOSI.G.R, 2007 : « La mobilisation des ressources propres locales en Afrique ». CERDI, article communiqué au Forum «Décentralisation et gouvernance locale » des Nations Unis, Vienne 27-29 juin 2007.

21. CHARLOT.S, SCHMITT.B, 1999 : «Infrastructures publiques et croissance des régions françaises ». UMR INRA-ENESED.

22. CONAN.M, 2008 : «Gaston Jèze et l'utilité de la dépense publique: l'élaboration d'une théorie générale des dépenses publiques », La Revue du Trésor, n², p 159-165, Février 2008.

23. CRAFTS.N, 1992: « Productivity Growth Reconsidered » Economic policy, p. 387-426.

24. DEBRUYNE.M, 2016: «Fiscalité, Relation de Confiance et Responsabilité Sociale de l'Entreprise », Revue de Management et de Stratégie, (5:2), pp 103-119, Université Lille III.

25. ELKTIRI.M, 1997: «Fiscalité et développement au Maroc ». Editions Magrébines 1982, Université Michigan, 215 P.

26. GLACHANT.J, 1992: « Cœur de croissance dans une économie loglinéaire », mimeo-MAD.

27. GARCIA-MILA.T, McGUIRE.T, PORTER.R.H, 1996: « The Effect of Public Capital in State-Level Production Functions », The 
Review of Economics and Statistics, Vol 78, n²1, p 177-180. Feb 1996.

28. GUELLEC.D, 1992: «Croissance endogène : les principaux mécanismes », Economie et prévision, 106. P 41-50.

29. GUELLEC.D, RALLE.P, 1993: «Innovation, propriété industrielle, croissance », Revue Economique 44 (2), mars. P 319-344.

30. GUPTA.S, TAREQ.S, 2008: «Mobilisation des recettes »; Finances et Développement, Septembre 2008.

31. HENIN.P.Y, CHATEAU.J, 1992: «Ecarts conjoncturels et croissance dans six économies de l’OCDE », mimeo, Cepremap, Octobre.

32. HENIN.P.Y, RALLE.P, 1993 : «Les nouvelles théories de la croissance : quelques apports pour la politique économique », Revue Economique, p 75-100.

33. HORUSITZKY.P, 2013: «L'Etat et les politiques publiques Enjeux, acteurs et dispositifs- 2d éd. Catégorie A et B. Dunod, 4 septembre 2013.

34. ITRIAGO.D, 2011 : «Décider de son développement: la fiscalité pour combattre la pauvreté », Posséder développement, Rapport de Recherche d'OXFAM, Septembre 2011.

35. JEZE.G, 1936 : «Cours de Finances Publiques 1935-1936 » Université de Pris, Faculté de droit, Ed. Librairie générale de droit et de jurisprudence.

36. JOHNTON.D.J, 2002: « Fiscalité et Croissance ». L’observateur de l'OCDE, N²30.

37. KOKKE.M, WEYZIG.F, 2008 : «Fiscalité et financement du développement », Commerce \& Investissement, Document d’information de SOMO (Centre de Recherche des Multinationales », Octobre 2008.

38. LEFEBRE. P, 2002 : «Comment améliorer la mobilisation des ressources? par des taxes intelligentes, des dépenses productives ; une perspective de long terme, de la transparence et de l'information ». Ecole des Sciences de la Gestion, Université du Québec à Montréal.

39. LENON.G, 2012: «Fiscalité et Développement ». L’observateur de l’OCDE, l'annuel 2012.

40. LUCAS.R, 1988: « On the Mechanics of Economics Development », Journal of Monetary Economics, vol 22, num 3-42.

41. LYNDE.C, RICHMOND.J, 1992: « The Role of Public Capital in Production », Review of Economics and Statistics, p 37-44. 
42. MALINVAUD.E, 1993: «Regard d'un ancien sur les nouvelles théories de la croissance », Revue Economique, 44(2), mars, p 171188.

43. MOUMMI.A, 2012 : « La mobilisation des ressources fiscales et son impact sur les niveaux de vie des ménages : cas du Niger », Working Paper Series, n¹65, Banque Africaine du Développement, Décembre 2012.

44. OCDE, 2000: «Liens entre la Politique Economique et la Croissance : Constatations au Niveau International », Perspectives Economiques de l'OCDE 68, p 155-178.

45. OCDE, 2009: «Objectifs Croissance: Chapitre-5: Fiscalité et croissance économique », Réformes Economiques, n5, 2009/1.Ed de l'OCDE.

46. OUALI.S, 2007: « Réformes et libéralisation des investissements au Maroc », OCDE.

47. REBELO.S, 1991: «Long Run Policy Analysis and Long Run Growth », Journal of Political Economy, 99, p 500-521.

48. ROMER.P, 1986: «Increasing Returns and Long Run Growth», The Journal of Political Economy, vol 94, num 5, p 1002-1037, October 1986.

49. ROY.W, 2004: «L'investissement public dans les infrastructures de transport est-il source de croissance endogène? », Mangement and Policy Studies Seminar (MAPS) on Role of Rail Transport in the National Productivity, UIC, mars 2004, Tunis.

50. SOLOW.R, 1992: « Policies for Economic Growth», De Economist, 140, p-15.

51. STIGLITZ.J, 2012 : «Le prix de l’inégalité ». Edition Norton New York, les liens qui libèrent pour la traduction française, sep 2012.

52. TANZI.V, ZEE.H, 2001: «Une politique fiscale pour les pays en développement », Dossiers Economiques, n²7, FMI, mars 2001.

53. TATOM.J, 2007: « Is tax policy retarding Growth in Morocco? ». Networks financial Institute, working paper $n^{\circ}$ 2007-WP-27.

54. TSASA VANGU.J-P.K, 2012 : «Dérivation du modèle basique de Barro: Approche par l’optimisation dynamique non stochastique », Laboratoire d'Analyse-Recherche en Economie Quantitative (LAREQ) vol 1, num 005, mars 2012.

55. VALENDUC.C, 2011 : «Quelle structure des prélèvements pour quelle croissance? ». Les Cahiers, le cercle des économistes, PUF, Ed. Descartes \& Cie.

56. VEGANZONES.M.A, 2000 : «Infrastructures, investissement et croissance: un bilan de dix années de recherches », CERDI, Clermont Ferrand, Janvier 2000. 
57. WANG.Y-C, 2014: «Evidence of public capital spillovers and endogenous in Taiwan », Economic Modeling, $n^{\circ} 39$, p 314-321, Mars 2014.

58. WEBER.A, 2011 : «Estimation de la Production Potentielle au Maroc », Document de travail, FMI, Février 2011.

\section{Annexe:}

System: UNTITLED

Estimation Method: Least Squares

Date: 12/16/16 Time: 18:02

Sample: 19802015

Included observations: 36

Total system (balanced) observations 72

$\begin{array}{lc} & \text { Coefficient } \\ \mathrm{C}(1) & 1.469612 \\ \mathrm{C}(2) & 0.205846 \\ \mathrm{C}(3) & 0.618191 \\ \mathrm{C}(4) & 0.844439 \\ \mathrm{C}(5) & 4.997295 \\ \mathrm{C}(6) & 0.890903 \\ \mathrm{C}(7) & 0.296158 \\ \text { residual covariance } & 5.86 \mathrm{E}-07\end{array}$

$\begin{array}{lll}\text { Std. Error } & \text { t-Statistic } & \text { Prob. } \\ 0.711235 & 2.066282 & 0.0428 \\ 0.081667 & 2.520556 & 0.0142 \\ 0.055892 & 11.06054 & 0.0000 \\ 0.050094 & 16.85717 & 0.0000 \\ 0.832752 & 6.000941 & 0.0000 \\ 0.115765 & 7.695781 & 0.0000 \\ 0.059126 & 5.008964 & 0.0000\end{array}$

Determinant residual covariance

Equation: $\mathrm{y}=\mathrm{C}(1)+\mathrm{C}(2) * \mathrm{k}_{1}+\mathrm{C}(3) * \mathrm{k}_{2}+\mathrm{C}(4) * \mathrm{l}$

Observations: 36

$\begin{array}{ccc}\text { R-squared } & 0.99879 & \text { Mean dependent var12.74636 } \\ \text { Adjusted R-squared } & 0.99867 & \text { S.D. dependent var } 0.408058 \\ \text { S.E. of regression } & 0.01483 & \text { Sum squared resid } 0.007037 \\ \text { Durbin-Watson stat } & 2.383268 & \end{array}$

Equation: $\mathrm{k}_{1}=\mathrm{C}(5)+\mathrm{C}(6) * \mathrm{y}+\mathrm{C}(7) * \mathrm{~T}$

Observations: 36

$\begin{array}{cc}\text { R-squared } & 0.86040 \\ \text { Adjusted R-squared } & 0.85194 \\ & \\ \text { S.E. of regression } & 0.057896 \\ \text { Durbin-Watson stat } & 2.168204\end{array}$

\begin{tabular}{cc}
\multicolumn{2}{c}{$\begin{array}{c}\text { Mean dependent } \\
\text { var }\end{array}$} \\
$\begin{array}{cc}\text { S.D. dependent } \\
\text { var }\end{array}$ & 0.150464364 \\
$\begin{array}{c}\text { Sum squared } \\
\text { resid }\end{array}$ & 0.110615
\end{tabular}




\section{Y Residuals}

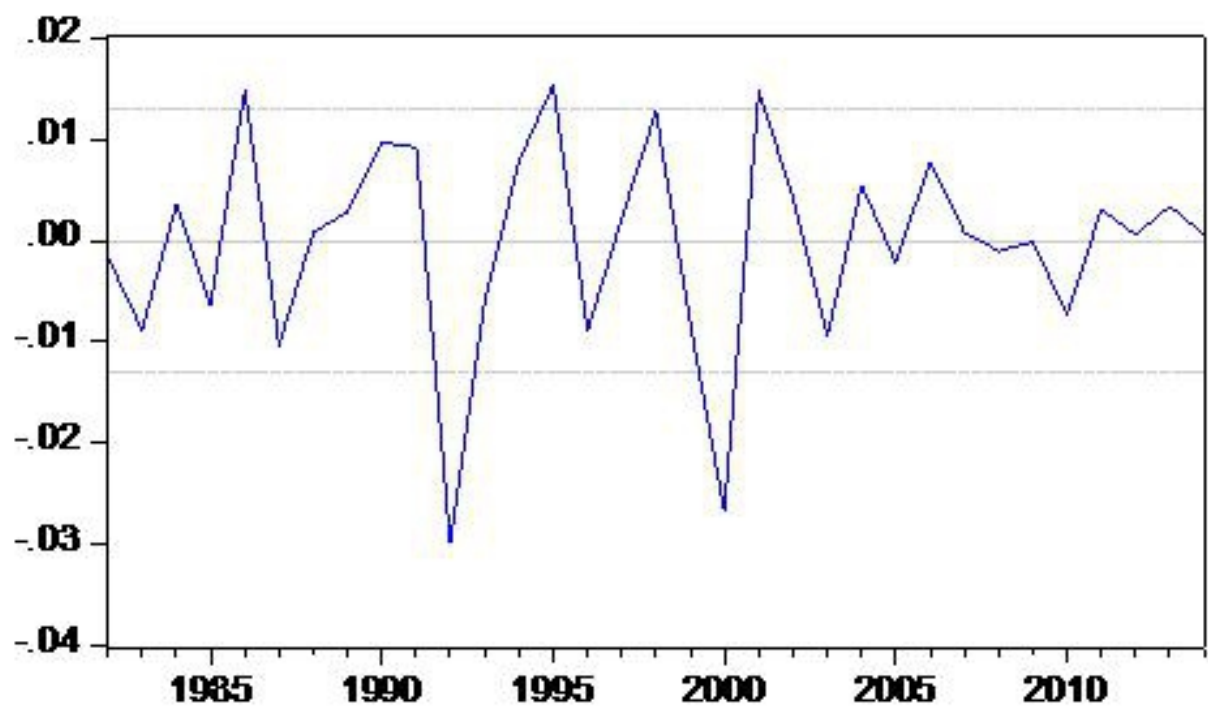

K1 Residuals

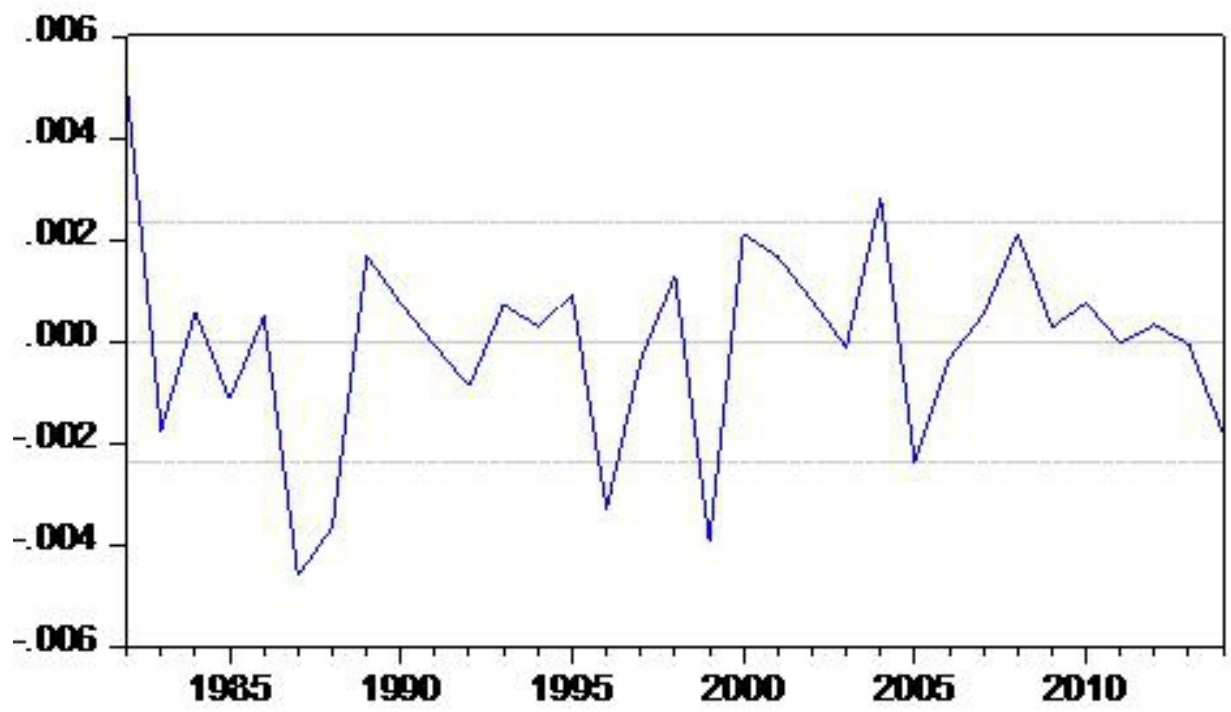

